\title{
COMPUTATION OF HOMOLOGY AND COHOMOLOGY GROUPS OF A METACYCLIC GROUP AND A FACTOR GROUP OF THE HEISENBERG GROUP
}

\author{
Subrata Majumdar and Nasima Akhter \\ Department of Mathematics \\ University of Rajshahi, Rajshahi-6205, Bangladesh \\ Email: nasima.math.ru@gmail.com
}

Received 15.7.08 Accepted 16.7.2011

\begin{abstract}
Free resolutions for a metacyclic group $M$ and a factor group $G$ of the Heisenberg group from their presentations constructed by solving system of linear equations over the integral group ring and determined the homology and cohomology groups. The method is straightforward, and the free resolutions are explicitly expressed in terms of the free generators. The resolutions yield the homology and the cohomology groups immediately.
\end{abstract}

Keywords: Group presentation, Metacyclic group, Heisenberg group, Free resolution, Huebschmann perturbation method, Homology, Cohomology.

\section{Introduction}

We have computed the homology and the cohomology groups of a metacyclic group $\mathrm{M}$ and a factor group $\mathrm{G}$ of the Heisenberg group by constructing free resolutions. The Heisenberg group $\mathrm{H}_{3}$ is the multiplicative group of the matrices $\left(\begin{array}{lll}1 & x & z \\ 0 & 1 & y \\ 0 & 0 & 1\end{array}\right)$ where $\mathrm{x}, \mathrm{y}, \mathrm{z}$ are real numbers ([22], p.467). Huebschmann[9] has determined the cohomology rings of $\mathrm{G}$ using the sophisticated and complicated machinery of perturbation theory developed by him. He has also dealt with the $\bmod p$ cohomology of $M$ [11]. To construct our resolutions we do not need to use any sophisticated machinery such as Künneth relations, spectral sequence and perturbation theory of homological algebra and Identity Theorem of combinatorial group theory. The "rewriting systems" of Groves [5] and Carbone [2] also have not been used. We have obtained our resolutions by extending Lyndon's partial resolution ([6], [12], [13]) through step by step solutions of systems of linear equations over the integral group rings. The details of the construction are given in [19]. Let $G=$ $F / R$ where $F$ is the free group on $\left\{x_{1}, \cdots, x_{m}\right\}$ and $R$ the normal closure of $\left\{r_{l}, \cdots, r_{n}\right\}$. Let $\pi: \mathbb{Z F} \rightarrow \mathbb{Z G}$ be the ring homomorphism which extends the canonical homomorphism from $F$ onto $G$, and let $\pi\left(x_{i}\right)=h_{i}$. Then Lyndon's partial free $\mathbb{Z} \mathrm{G}$ resolution of $\mathbb{Z}$ is the following: 
where

$Y_{0}$ is a free right $\mathbb{Z} \mathrm{G}$-module on $\left\{\alpha_{1}, \cdots, \alpha_{m}\right\}$,

$Y_{l}$ is a free right $\mathbb{Z} \mathrm{G}$-module on $\left\{\beta_{l}, \cdots, \beta_{n}\right\}$,

and $\varepsilon, d_{0}, d_{1}$ are $\mathbb{Z} G$-homomorphisms given by

$\varepsilon(g)=1$, for all $g \in G$

$d_{0}\left(\alpha_{i}\right)=h_{i}-1, i=1, \cdots, m$,

$d_{l}\left(\beta_{j}\right)=\sum_{i=1}^{m} a_{i} \pi\left(\frac{\partial r_{j}}{\partial x_{i}}\right), j=1, \cdots n$, Here $\frac{\partial r_{j}}{\partial x_{i}}$ is the Fox derivative [4] of $r_{j}$ with respect to $x_{i}$ and is defined by

$$
r-1=\sum_{i=1}^{n}\left(x_{i}-1\right) \frac{\partial r_{j}}{\partial x_{i}}, j=1, \cdots n
$$

For the extension of this partial resolution, the first set of equations is obtained by equating the right hand side expression for the map $d_{l}$ in $(\mathrm{P})$. The solutions of this set of equations is the kernel of $d_{1}$. From these solutions we can read off the generators of the next term $Y_{2}$ and the next homomorphism $d_{2}$ in the extension of (P) such that

$\operatorname{Im} d_{2}=\operatorname{Ker} d_{1}$.

Similarly, we can get the next term $Y_{3}$ and the next homomorphism $d_{3}$ in the extension from the set of equations obtained from $d_{2}$ as in the case of $d_{l}$. An extension of (P) to a full resolution is obtained by continuing this process.

As the terms of this resolution are free modules on explicitly given generators and the homomorphisms are explicitly defined on these generators, the resolutions immediately yield the homology and the cohomology groups with arbitrary coefficient modules. These have been determined and the integral homology and the cohomology groups have been explicitly calculated. One of the objectives in choosing these two groups is to exhibit the relative simplicity of our method in comparison to that of Huebschmann. While the latter method is applicable to a wide class of groups including nilpotent groups of class 2, our method too has been applied to different classes of groups ([1], [14], [15], [16], [17], [18], [20], [21]) and is applicable to many others.

\section{Determination of Homology and Cohomology of a factor group of the Heisenberg group}

The Group $G$ considered here is given by

generators : $h_{1}, h_{2}, h_{3}$;

relations : $\left[h_{1}, h_{2}\right]=h_{3},\left[h_{2}, h_{3}\right]=1=\left[h_{1}, h_{3}\right], h_{3}^{l}=1$, where $l \in \mathbb{N}$ 
Then $G$ can be viewed as a central extension of $\mathbb{Z}_{l}$ by $\mathbb{Z} \times \mathbb{Z}, \mathbb{Z}_{l}$ is a cyclic group of order $l$. Also we can see that $G$ is a factor group of Heisenberg group $H$ with generators $: h_{1}, h_{2}$, $h_{3}$; relations : $\left[h_{1}, h_{2}\right]=h_{3},\left[h_{2}, h_{3}\right]=1=\left[h_{1}, h_{3}\right], h_{3}^{l}=1$.

Thus $G=F / R$, where $F$ is the free group generated by $x_{1}, x_{2}, x_{3}$ and $R$ is the normal subgroup of $F$ generated by $r_{1}, r_{2}, r_{3}, r_{4}$ where

$$
r_{1}=x_{3}^{-1} x_{2}^{-1} x_{1}^{-1} x_{2} x_{1}, r_{2}=x_{2}^{-1} x_{3}^{-1} x_{2} x_{3}, r_{3}=x_{1}^{-1} x_{3}^{-1} x_{1} x_{3}, r_{4}=x_{3}^{1} .
$$

Then the fox derivatives are:

$$
\begin{array}{llll}
\frac{\partial r_{1}}{\partial x_{1}}=-x_{1}^{-1} x_{2} x_{1}+1, & \frac{\partial r_{1}}{\partial x_{2}}=-x_{2}^{-1} x_{1}^{-1} x_{2} x_{1}+x_{1}, & & \frac{\partial r_{1}}{\partial x_{3}}=-r_{1}, \\
\frac{\partial r_{2}}{\partial x_{1}}=0, & \frac{\partial r_{2}}{\partial x_{2}}=-r_{2}+x_{3}, & \frac{\partial r_{2}}{\partial x_{3}}=-x_{3}^{-1} x_{2} x_{3}+1, \\
\frac{\partial r_{3}}{\partial x_{1}}=-r_{3}+x_{3}, & \frac{\partial r_{3}}{\partial x_{2}}=0, & \frac{\partial r_{3}}{\partial x_{3}}=-x_{3}^{-1} x_{1} x_{3}+1, \\
\frac{\partial r_{4}}{\partial x_{1}}=0, & \frac{\partial r_{4}}{\partial x_{2}}=0, & \frac{\partial r_{4}}{\partial x_{3}}=-x_{3}^{l-1}+\ldots \ldots+1,
\end{array}
$$

Let $\pi: \mathbb{Z F} \rightarrow \mathbb{Z G}$ be the ring homomorphism induced by the canonical homomorphism of $F$ onto $G$ with $R$ as the kernel. Let $\pi\left(x_{i}\right)=h_{i}, i=1,2,3$. Then,
$\pi\left(\frac{\partial r_{1}}{\partial x_{1}}\right)=-h_{1}^{-1} h_{2} h_{1}+1$
$\pi\left(\frac{\partial r_{1}}{\partial x_{2}}\right)=h_{1^{-}} h_{3}$
$\pi\left(\frac{\partial r_{1}}{\partial x_{3}}\right)=-1$
$\pi\left(\frac{\partial r_{2}}{\partial x_{1}}\right)=0$
$\pi\left(\frac{\partial r_{2}}{\partial x_{2}}\right)=h_{3}-1$
$\pi\left(\frac{\partial r_{2}}{\partial x_{3}}\right)=1-h_{2}$
$\pi\left(\frac{\partial r_{3}}{\partial x_{1}}\right)=h_{3}-1$
$\pi\left(\frac{\partial r_{3}}{\partial x_{2}}\right)=0$
$\pi\left(\frac{\partial r_{3}}{\partial x_{3}}\right)=1-h_{1}$
$\pi\left(\frac{\partial r_{4}}{\partial x_{1}}\right)=0$
$\pi\left(\frac{\partial r_{4}}{\partial x_{2}}\right)=0$
$\pi\left(\frac{\partial r_{4}}{\partial x_{3}}\right)=h_{3}^{l-1}+\ldots \ldots+1$,

\section{Theorem 1.1}

The following is a free $\mathbb{Z} G$-resolution of $\mathbb{Z}$ :

$$
Y_{1} \stackrel{d_{2}}{\rightarrow} Y_{1} \stackrel{d_{3}}{\rightarrow} Y_{1} \stackrel{d_{2}}{\rightarrow} Y_{1} \stackrel{d_{2}}{\rightarrow} Y_{1} \stackrel{d_{0}}{\rightarrow} \mathbb{Z} \stackrel{\varepsilon}{\rightarrow} \mathbb{Z} \rightarrow 0
$$

where

$Y_{0}$ is a right $\mathbb{Z} G$-module free on $\alpha_{1}, \alpha_{2}, \alpha_{3}$; 
$Y_{1}$ is a right $\mathbb{Z} G$-module free on $\beta_{1}, \beta_{2}, \beta_{3}, \beta_{4}$;

and $\varepsilon, d_{0}, d_{1}, d_{2}, d_{3}$ are the $\mathbb{Z} G$-homomorphisms and given by

$\varepsilon(g)=1$, for all $g \in G$

$d_{0}\left(\alpha_{i}\right)=h_{i}-1, i=1,2,3$,

$d_{1}\left(\beta_{j}\right)=\sum_{i=1}^{3} \alpha_{i} \pi\left(\frac{\partial r_{j}}{\partial x_{i}}\right), j=1,2,3,4$

$d_{2}\left(\beta_{1}\right)=\beta_{1}\left(h_{3}-1\right)-\beta_{2}\left(h_{1}-h_{2}\right)+\beta_{3}\left(1-h_{2} h_{3}\right)$

$d_{2}\left(\beta_{2}\right)=\beta_{2}\left(h_{3}^{l-1}+\ldots \ldots+1\right)+\beta_{4}\left(h_{2}-1\right)$,

$d_{2}\left(\beta_{3}\right)=\beta_{3}\left(h_{3}{ }^{l-1}+\ldots \ldots+1\right)+\beta_{4}\left(h_{1}-1\right)$,

$d_{2}\left(\beta_{4}\right)=\beta_{4}\left(h_{3}-1\right)$,

$d_{3}\left(\beta_{1}\right)=\beta_{1}\left(h_{3}{ }^{l-1}+\ldots \ldots+1\right)+\beta_{2}\left(h_{1}-h_{3}\right)+\beta_{3}\left(1-h_{2} h_{3}\right)-\beta_{4}$,

$d_{3}\left(\beta_{2}\right)=\beta_{2}\left(h_{3}-1\right)+\beta_{4}\left(1-h_{2}\right)$,

$d_{3}\left(\beta_{3}\right)=\beta_{3}\left(h_{3}-1\right)+\beta_{4}\left(1-h_{1}\right)$,

$d_{3}\left(\beta_{4}\right)=\beta_{4}\left(h_{3}^{l-1}+\ldots \ldots+1\right)$.

\section{Proof}

By $(\mathrm{P})$ it is sufficient to verify the exactness of the sequence only at the first, second and third $Y_{l}$ 's from the right.

Exactness at $\underline{Y}_{1}$ (first from the right)

$$
\begin{aligned}
d_{1} d_{2}\left(\beta_{1}\right)= & d_{1}\left[\beta_{1}\left(h_{3}-1\right)-\beta_{2}\left(h_{1}-h_{3}\right)-\beta_{3}\left(1-h_{2} h_{3}\right)\right] \\
= & \left(\alpha_{1}\left(1-h_{2} h_{3}\right)+\alpha_{2}\left(h_{1}-h_{3}\right)-\alpha_{3}\right)\left(h_{3}-1\right)-\left(\alpha_{2}\left(h_{3}-1\right)+\alpha_{3}\left(1-h_{2}\right)\right)\left(h_{1}-h_{3}\right) \\
& \left.-\left(\alpha_{1}\left(h_{3}-1\right)+\alpha_{3}\left(1-h_{1}\right)\right)\left(1-h_{2} h_{3}\right)\right) \\
= & 0,
\end{aligned}
$$

Similarly, one can easily show that, $d_{1} d_{2}\left(\beta_{2}\right)=0=d_{1} d_{2}\left(\beta_{3}\right)=d_{1} d_{2}\left(\beta_{4}\right)$.

$\operatorname{Ker} d_{1} \subset \operatorname{Im} d_{2}$.

Conversely, let $d_{1}\left(\beta_{1} \gamma_{1}+\beta_{2} \gamma_{2}+\beta_{3} \gamma_{3}+\beta_{4} \gamma_{4}\right)=0$, for some $\gamma_{i} \in \mathbb{Z} G, i=1,2,3,4$.

Then $\left[\alpha_{1}\left(1-h_{2} h_{3}\right)+\alpha_{2}\left(h_{1}-h_{3}\right)-\alpha_{3}\right] \gamma_{1}+\left[\alpha_{2}\left(h_{3}-1\right)+\alpha_{3}\left(1-h_{2}\right)\right] \gamma_{2}$

$$
+\left[\alpha_{1}\left(h_{3}-1\right)+\alpha_{3}\left(1-h_{1}\right)\right] \gamma_{3}+\alpha_{3}\left(h_{3}{ }^{l-1}+\ldots \ldots+1\right) \gamma_{4}=0 .
$$

Since, $Y_{0}$ is free on $\alpha_{1}, \alpha_{2}, \alpha_{3}$, this implies that

$$
\begin{aligned}
& \left(1-h_{2} h_{3}\right) \gamma_{1}+\left(h_{3}-1\right) \gamma_{3}=0 \\
& \left(h_{1}-h_{3}\right) \gamma_{1}+\left(h_{3}-1\right) \gamma_{2}=0 \\
& -\gamma_{1}+\left(1-h_{2}\right) \gamma_{2}+\left(1-h_{1}\right) \gamma_{3}+\left(h_{3}{ }^{l-1}+\ldots \ldots+1\right) \gamma_{4}=0
\end{aligned}
$$


Multiplying (1) by $h_{3}{ }^{l-1}+\ldots \ldots+1$, we have

$\left(h_{3}^{l-1}+\ldots \ldots+1\right)\left(1-h_{2}\right) h_{3} \gamma_{1}=0$. Since the order of $h_{2}$ is not finite,

$\left(h_{3}^{l-1}+\ldots \ldots+1\right) \gamma_{1}=0$. Hence by Proposition 1 of [14]

$\gamma_{1}=\left(h_{3}-1\right) \gamma^{\prime}{ }_{1}$ for some $\gamma^{\prime}{ }_{1} \in \mathbb{Z} G$.

Put the value of $\gamma_{1}$ in (2), we get

$$
\left(h_{3}-1\right)\left[\left(h_{1}-h_{2}\right) \gamma_{1}^{\prime}+\gamma_{2}\right]=0 \text {. Therefore, }
$$

$\left(h_{l}-h_{3}\right) \gamma^{\prime}{ }_{1}+\gamma_{2}=\left(h_{3}{ }^{l-1}+\ldots \ldots+1\right) \gamma_{2}^{\prime}$, for some $\gamma_{2}{ }_{2} \in \mathbb{Z} G$, by Proposition 2 of [14].

$$
\therefore \gamma_{2}=\left(h_{3}^{l-1}+\ldots \ldots+1\right) \gamma_{2}^{\prime}-\left(h_{1}-h_{3}\right) \gamma_{1}^{\prime} \text {. }
$$

Substituting for $\gamma_{1}$ in (1) and applying Proposition 2 of [14] we have, $\gamma_{3}=\left(h_{3}^{l-1}+\ldots \ldots+1\right) \gamma_{3}^{\prime}-\left(1-h_{2} h_{3}\right) \gamma_{1 \text {. }}^{\prime}$

Now putting the value of $\gamma_{1}, \gamma_{2}$ and $\gamma_{3}$ in (3), we get,

$$
\left(h_{3}^{l-1}+\ldots \ldots+1\right)\left[\left(1-h_{2}\right) \gamma_{2}^{\prime}+\left(1-h_{1}\right) \gamma_{3}^{\prime}+\gamma_{4}\right]=0 \text {. }
$$

Thus, $\gamma_{4}=\left(h_{3}-1\right) \gamma^{\prime}{ }_{4}+\left(h_{2}-1\right) \gamma_{2}{ }_{2}+\left(h_{1}-1\right) \gamma_{3}^{\prime}$, for some $\gamma^{\prime} \in \mathbb{Z} G$.

Hence $\beta_{1} \gamma_{1}+\beta_{2} \gamma_{2}+\beta_{3} \gamma_{3}+\beta_{4} \gamma_{4}$

$$
\begin{aligned}
& =\left\{\beta_{1}\left(h_{3}-1\right)-\beta_{2}\left(h_{1}-h_{3}\right)-\beta_{3}\left(1-h_{2} h_{3}\right)\right\} \gamma^{{ }_{1}}{ }_{1}+\left\{\beta_{2}\left(h_{3}{ }^{l-1}+\ldots \ldots+1\right)+\beta_{4}\left(h_{2}-1\right)\right\} \gamma^{\prime}{ }_{2} \\
& +\left\{\beta_{3}\left(h_{3}{ }^{l-1}+\ldots \ldots+1\right)+\beta_{4}\left(h_{1}-1\right)\right\} \gamma^{\prime}{ }_{3}+\beta_{4}\left(h_{3}-1\right) \gamma_{4}^{\prime} \\
& =d_{2}\left(\beta_{1} \gamma^{\prime}{ }_{1}+\beta_{2} \gamma^{\prime}{ }_{2}+\beta_{3} \gamma^{\prime}{ }_{3}+\beta_{4} \gamma^{\prime}{ }_{4}\right) \subset \operatorname{Im} d_{2} .
\end{aligned}
$$

$\therefore \operatorname{Ker} d_{1}=\operatorname{Im} d_{2}$.

Exactness at $\boldsymbol{Y}_{1}$ (second from the right)

The kernel of $d_{2}$ contains the image of $d_{3}$ is obvious; so we only prove its converse.

Let $\beta_{1} \gamma_{1}+\beta_{2} \gamma_{2}+\beta_{3} \gamma_{3}+\beta_{4} \gamma_{4} \in \operatorname{Ker} d_{2}$,

Then $d_{2}\left(\beta_{1} \gamma_{1}+\beta_{2} \gamma_{2}+\beta_{3} \gamma_{3}+\beta_{4} \gamma_{4}\right)=0$.

$$
\text { i.e., } \begin{gathered}
{\left[\beta_{1}\left(h_{3}-1\right)-\beta_{2}\left(h_{1}-h_{3}\right)-\beta_{3}\left(1-h_{2} h_{3}\right)\right] \gamma_{1}+\left\{\beta_{2}\left(h_{3}{ }^{l-1}+\ldots \ldots+1\right)+\beta_{4}\left(h_{2}-1\right)\right\} \gamma_{2}} \\
+\left\{\beta_{3}\left(h_{3}{ }^{l-1}+\ldots \ldots+1\right)+\beta_{4}\left(h_{1}-1\right)\right\} \gamma_{3}+\beta_{4}\left(h_{3}-1\right) \gamma_{4}=0 .
\end{gathered}
$$

Since $\beta_{1}, \beta_{2}, \beta_{3}, \beta_{4}$, are linearly independent

$$
\begin{aligned}
& \left(h_{3}-1\right) \gamma_{1}=0 \\
& \left(h_{3}^{l-1}+\ldots \ldots+1\right) \gamma_{2}-\left(h_{1}-h_{3}\right) \gamma_{1}=0 \\
& \left(h_{3}{ }^{l-1}+\ldots \ldots+1\right) \gamma_{3}-\left(1-h_{2} h_{3}\right) \gamma_{1}=0 \\
& \left(h_{3}-1\right) \gamma_{4}+\left(h_{1}-1\right) \gamma_{3}+\left(h_{2}-1\right) \gamma_{2}=0 . \text { Thus, from the first equation, we have } \\
& \gamma_{1}=\left(h_{3}^{l-1}+\ldots \ldots+1\right) \gamma^{\prime}{ }_{1}, \text { for some } \gamma^{\prime}{ }_{1} \in \mathbb{Z} G, \text { by Proposition } 2 \text { of }[14] .
\end{aligned}
$$


Putting the value of $\gamma_{1}$ in the second and third equations and applying Proposition 1 of [14], we get

$$
\begin{aligned}
& \gamma_{2}=\left(h_{3}-1\right) \gamma_{2}{ }_{2}+\left(h_{1}-h_{3}\right) \gamma_{1}^{\prime} \\
& \gamma_{3}=\left(h_{3}-1\right) \gamma_{3}^{\prime}+\left(1-h_{2} h_{3}\right) \gamma_{1}^{\prime}, \text { for some } \gamma_{2}^{\prime}, \gamma_{3}^{\prime} \in \mathbb{Z} G \text { respectively. }
\end{aligned}
$$

Substituting for $\gamma_{2}$ and $\gamma_{3}$ in the last equation and an application of Proposition 2 of [14], gives

$$
\gamma_{4}=\left(h_{3}{ }^{l-1}+\ldots \ldots+1\right) \gamma^{\prime}{ }_{4}-\gamma^{\prime}{ }_{1}+\left(1-h_{2}\right) \gamma^{\prime}{ }_{2}+\left(1-h_{1}\right) \gamma^{\prime}{ }_{1} \text {, for some } \gamma_{4}^{\prime} \in \mathbb{Z} G \text {. }
$$

Therefore, $\beta_{1} \gamma_{1}+\beta_{2} \gamma_{2}+\beta_{3} \gamma_{3}+\beta_{4} \gamma_{4}=d_{3}\left(\beta_{1} \gamma^{\prime}{ }_{1}+\beta_{2} \gamma^{\prime}{ }_{2}+\beta_{3} \gamma^{\prime}{ }_{3}+\beta_{4} \gamma^{\prime}{ }_{4}\right) \in \operatorname{Im~d}_{3}$.

$\therefore \operatorname{Ker} d_{2}=\operatorname{Im} d_{3}$

\section{Exactness at $\boldsymbol{Y}_{1}$ (third from the right)}

By the definitions of $d_{2}$ and $d_{3}$

$\operatorname{Ker} d_{3} \subset \operatorname{Im} d_{2}$.

So let $\beta_{1} \gamma_{1}+\beta_{2} \gamma_{2}+\beta_{3} \gamma_{3}+\beta_{4} \gamma_{4} \subset \operatorname{Ker} d_{3}$, then $d_{3}\left(\beta_{1} \gamma_{1}+\beta_{2} \gamma_{2}+\beta_{3} \gamma_{3}+\beta_{4} \gamma_{4}\right)=0$,

i.e., $\beta_{1}\left(h_{3}{ }^{l-1}+\ldots\right.$ $.+1) \gamma_{1}+\beta_{2}\left(h_{1}-h_{3}\right) \gamma_{1}+\beta_{3}\left(1-h_{2} h_{3}\right) \gamma_{1}-\beta_{4} \gamma_{1}+\beta_{2}\left(h_{3}-1\right) \gamma_{2}+\beta_{4}\left(1-h_{2}\right) \gamma_{2}$

$$
+\beta_{3}\left(h_{3}-1\right) \gamma_{3}+\beta_{4}\left(1-h_{1}\right) \gamma_{3}+\beta_{4}\left(h_{3}{ }^{l-1}+\ldots \ldots+1\right) \gamma_{4}=0 .
$$

Since $Y_{1}$ is free on $\beta_{1}, \beta_{2}, \beta_{3}, \beta_{4}$, we have equations

$$
\begin{aligned}
& \left(h_{3}^{l-1}+\ldots \ldots+1\right) \gamma_{1}=0, \\
& \left(h_{1}-h_{3}\right) \gamma_{1}+\left(h_{3}-1\right) \gamma_{2}=0, \\
& \left(1-h_{2} h_{3}\right) \gamma_{1}+\left(h_{3}-1\right) \gamma_{3}=0, \\
& -\gamma_{1}+\left(1-h_{2}\right) \gamma_{2}+\left(1-h_{1}\right) \gamma_{3}+\left(h_{3}{ }^{l-1}+\ldots \ldots+1\right) \gamma_{4}=0 .
\end{aligned}
$$

From the first equation, by Proposition 1 of [14], we have

$$
\gamma_{1}=\left(h_{3}-1\right) \gamma_{1}^{\prime} \text {, for some } \gamma^{\prime}{ }_{1} \in \mathbb{Z} G \text {. }
$$

Substituting in the second equation and applying Proposition 1 of [14], we get

$$
\gamma_{2}=\left(h_{3}{ }^{l-1}+\ldots \ldots+1\right) \gamma_{2}^{\prime}-\left(h_{1}-h_{3}\right) \gamma_{1}^{\prime} \text {, for some } \gamma_{2}^{\prime} \in \mathbb{Z} G \text {. }
$$

Similarly, the third equation gives

$$
\gamma_{3}=\left(h_{3}^{l-1}+\ldots \ldots+1\right) \gamma_{3}^{\prime}-\left(1-h_{2} h_{3}\right) \gamma_{1}^{\prime} \text {, for some } \gamma_{3}^{\prime} \in \mathbb{Z} G \text {. }
$$

Substituting for $\gamma_{1}, \gamma_{2}$ and $\gamma_{3}$ in the last equation and applying Proposition 1 of [14], we get

$$
\gamma_{4}=\left(h_{3}-1\right) \gamma_{4}^{\prime}-\left(1-h_{2}\right) \gamma_{2}^{\prime}-\left(1-h_{1}\right) \gamma_{3}^{\prime} \text {, for some } \gamma_{4}^{\prime} \in \mathbb{Z} G \text {. }
$$

Thus, $\beta_{1} \gamma_{1}+\beta_{2} \gamma_{2}+\beta_{3} \gamma_{3}+\beta_{4} \gamma_{4}=d_{2}\left(\beta_{1} \gamma^{\prime}{ }_{1}+\beta_{2} \gamma^{\prime}{ }_{2}+\beta_{3} \gamma^{\prime}{ }_{3}+\beta_{4} \gamma^{\prime}{ }_{4}\right)$.

$$
\therefore \operatorname{Ker} d_{3}=\operatorname{Im} d_{2} \text {. }
$$


By the nature of the sequence we do not need to consider any more terms, and the proof of the theorem is complete.

\section{Homology Groups of $\boldsymbol{G}$}

Let $A$ be a left $\mathbb{Z} G$-module. The homology groups $H_{n}(G, A)$ are given by the homology of the complex:

$$
A^{4} \stackrel{\bar{d}_{2}}{\rightarrow} A^{4} \stackrel{\bar{d}_{3}}{\rightarrow} A^{4} \stackrel{\bar{d}_{2}}{\rightarrow} A^{4} \stackrel{\bar{d}_{2}}{\rightarrow} A^{4} \stackrel{\bar{d}_{0}}{\rightarrow} A \rightarrow 0
$$

Where $A^{n}$ stands for the direct sum of $n$ isomorphic copies of $A$ and the homomorphisms $\bar{d}_{0}, \bar{d}_{1}, \bar{d}_{2}, \bar{d}_{3}$ are induced by $d_{0}, d_{1}, d_{2}, d_{3}$ respectively and are given by

$$
\begin{aligned}
\bar{d}_{0}\left(a_{1}, a_{2}, a_{3}\right)= & \left(h_{1}-1\right) a_{1}+\left(h_{2}-1\right) a_{2}+\left(h_{3}-1\right) a_{3}, \\
\bar{d}_{1}\left(a_{1}, a_{2}, a_{3}, a_{4}\right)= & \left(\left(1-h_{2} h_{3}\right) a_{1}+\left(h_{3}-1\right) a_{3},\left(h_{1}-h_{3}\right) a_{1}+\left(h_{3}-1\right) a_{2},-a_{1}+\left(1-h_{2}\right) a_{2}\right. \\
& \left.+\left(1-h_{1}\right) a_{3}+\left(h_{3}^{l-1}+\ldots \ldots+1\right) a_{4}\right), \\
\bar{d}_{2}\left(a_{1}, a_{2}, a_{3}, a_{4}\right) & =\left(\left(h_{3}-1\right) a_{1},\left(h_{3}^{l-1}+\ldots \ldots+1\right) a_{2}-\left(h_{1}-h_{3}\right) a_{1},\left(h_{3}{ }^{l-1}+\ldots \ldots+1\right) a_{3}\right. \\
& \left.-\left(1-h_{2} h_{3}\right) a_{1},\left(h_{3}-1\right) a_{4}+\left(h_{1}-1\right) a_{3}+\left(1-h_{2}\right) a_{2}\right), \\
\bar{d}_{3}\left(a_{1}, a_{2}, a_{3}, a_{4}\right) & =\left(\left(h_{3}^{l-1}+\ldots \ldots+1\right) a_{1},\left(h_{3}-1\right) a_{2}+\left(h_{1}-h_{3}\right) a_{1},\left(h_{3}-1\right) a_{3}\right. \\
& \left.+\left(1-h_{2} h_{3}\right) a_{3},\left(h_{3}^{l-1}+\ldots \ldots+1\right) a_{4}+\left(1-h_{1}\right) a_{3}+\left(1-h_{2}\right) a_{2}-a_{1}\right)
\end{aligned}
$$

for all $a_{1}, a_{2}, a_{3}, a_{4} \in A$

When $A$ is a trivial $\mathbb{Z} G$-module $\mathbb{Z}$, the above homomorphisms of the complex reduce to the following:

$$
\begin{aligned}
& \bar{d}_{0}\left(a_{1}, a_{2}, a_{3}\right)=0, \\
& \bar{d}_{1}\left(a_{1}, a_{2}, a_{3}, a_{4}\right)=\left(0,0,-a_{1}+l a_{4}\right), \\
& \bar{d}_{2}\left(a_{1}, a_{2}, a_{3}, a_{4}\right)=\left(0, l a_{2}, l a_{3}, 0\right), \\
& \bar{d}_{3}\left(a_{1}, a_{2}, a_{3}, a_{4}\right)=\left(l a_{1}, 0,0, l a_{4}-a_{1}\right) .
\end{aligned}
$$

Therefore, in this case, we have

$$
\begin{aligned}
& \mathrm{H}_{0}(G, \mathbb{Z}) \cong \mathbb{Z} \\
& \mathrm{H}_{1}(G, \mathbb{Z})=\frac{\operatorname{Ker} \bar{d}_{0}}{\operatorname{lm} \bar{d}_{2}}=\frac{\left\{\left(a_{2}, a_{2}, a_{3}\right) \mid a_{2}, a_{2}, a_{3}, \in Z\right\}}{\left\{\left(0,0,-a_{2}+a_{4}\right) \mid a_{2}, a_{4} \in Z\right\}} \cong(x, y, z \mid z=0, l z=0) \cong \mathbb{Z}^{2} \\
& \mathrm{H}_{2}(G, \mathbb{Z})=\frac{\operatorname{Ker} \bar{d}_{2}}{\operatorname{lm} \bar{d}_{2}}=\frac{\left\{\left(a_{1}, a_{2}, a_{3}, a_{4}\right) \mid a_{2}=l a_{4}, a_{z}, a_{3}, a_{4} \in Z\right\}}{\left\{\left(0, l a_{2}+l a_{4}, 0\right) \mid a_{2}, a_{3} \in Z\right\}}=\frac{(x) \oplus(y) \oplus(z)}{(l y)+(l z)}
\end{aligned}
$$




$$
\begin{aligned}
& \cong(x, y, z \mid l y=0, l z=0) \cong \mathbb{Z} \oplus \mathbb{Z}_{l} \oplus \mathbb{Z}_{l} \\
& \mathrm{H}_{3}(G, \mathbb{Z}) \frac{\operatorname{Ker} \bar{d}_{2}}{\operatorname{lm} \bar{d}_{3}}=\frac{\left\{\left(a_{1}, a_{2}, a_{3}, a_{4}\right) \mid a_{2}=0=a_{3} ; a_{3}, a_{2}, a_{2}, a_{4} \in Z\right\}}{\left\{\left(l a_{2}, 0,0, l a_{4}-a_{2}\right) \mid a_{2}, a_{4} \in Z\right\}} \\
& =\frac{(x) \oplus(y)}{(l x-y)+(l y)}, \text { writing } x=(1,0) \text { and } y=(0,1) . \\
& =(x, y \mid y=l x, l y=0)=\left(\mathrm{x} \mid l^{2} x=0\right) \cong \mathbb{Z}_{l}^{2} \\
& =\mathrm{H}_{4}(\mathrm{G}, \mathbb{Z})=\frac{\operatorname{Ker}_{2}}{\operatorname{lm} \bar{d}_{3}}=\frac{\left\{\left(a_{2}, a_{2}, a_{3}, a_{4}\right) \mid l a_{2}=0, a_{2}=l a_{4} ; a_{2}, a_{2}, a_{3}, a_{4} \in Z\right\}}{\left\{\left(0, l a_{2}, l a_{3}, 0\right) \mid a_{2}, a_{3} \in Z\right\}}=\frac{(x) \oplus(y)}{(l x)+(l y)} \\
& \cong(x, y \mid l x=0, l y=0)=\left(x \mid l^{2} x=0\right) \cong \mathbb{Z} l \oplus \mathbb{Z}_{l}
\end{aligned}
$$

Thus $\mathrm{H}_{2 n-1}(\mathrm{G}, \mathbb{Z}) \cong \mathbb{Z}^{2}, \mathrm{n} \geq 2$.

$\mathrm{H}_{2 n}(\mathrm{G}, \mathbb{Z}) \cong \mathbb{Z}_{l} \oplus \mathbb{Z}_{l}, \mathrm{n} \geq 2$.

\section{Cohomology Groups of $G$}

Let $\mathrm{A}$ be a right $\mathbb{Z} G$-module, then the cohomology groups $H^{n}(G, A)$ are given by the homology of the complex:

$$
\leftarrow A^{4} \stackrel{d_{2}^{*}}{\leftarrow} A^{4} \stackrel{d_{3}^{*}}{\leftarrow} A^{4} \stackrel{d_{2}^{*}}{\leftarrow} A^{4} \stackrel{d_{1}^{*}}{\leftarrow} A^{4} \stackrel{d_{0}^{*}}{\leftarrow} A \leftarrow 0
$$

where $A^{n}$ is the direct sum of $n$ isomorphic copies of $A$ and the homomorphisms $d_{0}^{*}, d_{1}^{*}, d_{2}^{*}, d_{3}^{*}$ are given by $d_{0}, d_{1}, d_{2}, d_{3}$ respectively and given by

$$
\begin{aligned}
& d_{0}^{*}(a)=\left(a\left(h_{1}-1\right), a\left(h_{2}-1\right), a\left(h_{3}-1\right)\right) \text {, } \\
& d_{1}^{*}\left(a_{1}, a_{2}, a_{3}\right)=\left(a_{1}\left(1-h_{2} h_{3}\right)+a_{2}\left(h_{1}-h_{3}\right)-a_{3}, a_{2}\left(h_{3}-1\right)+a_{3}\left(1-h_{2}\right),\right. \\
& \left.a_{1}\left(h_{3}-1\right)+a_{3}\left(1-h_{1}\right), a_{3}\left(h_{3}^{l-1}+\ldots \ldots+1\right)\right), \\
& d_{2}^{*}\left(a_{1}, a_{2}, a_{3}, a_{4}\right)=\left(a_{1}\left(h_{3}-1\right)-a_{2}\left(h_{1}-h_{3}\right)-a_{3}\left(1-h_{2} h_{3}\right), a_{2}\left(h_{3}{ }^{l-1}+\ldots \ldots+1\right)\right. \\
& \left.+a_{4}\left(h_{2}-1\right), a_{3}\left(h_{3}^{l-1}+\ldots \ldots+1\right)+a_{4}\left(h_{1}-1\right), a_{4}\left(h_{3}-1\right)\right), \\
& d_{3}^{*}\left(a_{1}, a_{2}, a_{3}, a_{4}\right)=\left(a_{1}\left(h_{3}^{l-1}+\ldots \ldots+1\right)+a_{2}\left(h_{1}-h_{3}\right)+a_{3}\left(1-h_{1} h_{2}\right)-a_{4}, a_{2}\left(h_{3}-1\right)\right) \\
& \left.+a_{4}\left(1-h_{2}\right), a_{3}\left(h_{3}-1\right)+a_{4}\left(1-h_{1}\right), a_{4}\left(h_{3}{ }^{l-1}+\ldots \ldots+1\right)\right) .
\end{aligned}
$$

When $A$ is a trivial $\mathbb{Z} G$-module $\mathbb{Z}$, the above homomorphisms of the complex reduce to the following:

$$
\begin{aligned}
& d_{0}^{*}(a)=(0,0,0), \\
& d_{1}^{*}\left(a_{1}, a_{2}, a_{3}\right)=\left(-a_{3}, 0,0, l a_{3}\right), \\
& d_{2}^{*}\left(a_{1}, a_{2}, a_{3}, a_{4}\right)=\left(0, l a_{2}, l a_{3}, 0\right), \\
& d_{3}^{*}\left(a_{1}, a_{2}, a_{3}, a_{4}\right)=\left(l a_{1}-a_{4}, 0,0, l a_{4}\right) .
\end{aligned}
$$


Therefore, in this case, we have

$\mathrm{H}^{0}(\mathrm{G}, \mathbb{Z}) \cong \mathbb{Z}$

$\mathrm{H}^{1}(\mathrm{G}, \mathbb{Z})=\frac{\operatorname{Kerd}_{1}^{*}}{\operatorname{lm} d_{0}^{*}}=\frac{\left\{\left(a_{2}, a_{2}, 0\right) \mid a_{2}, a_{2} \in Z\right\}}{\{0,0,0\}} \cong \mathbb{Z} \oplus \mathbb{Z}$

$\mathrm{H}^{2}(\mathrm{G}, \mathbb{Z})=\frac{\operatorname{Kerd}_{2}^{*}}{l m d_{1}^{*}}=\frac{\left\{\left(a_{2}, 0,0 a_{4}\right) \mid a_{2}, a_{4} \in Z\right\}}{\left.\left\{-a_{3}, 0,0, l a_{3}\right) \mid a_{3} \in Z\right\}} \cong \frac{(x) \oplus(y)}{(-x+l y)} \cong(x, y \mid x=l y) \cong \mathbb{Z}_{l}^{2}$

$\mathrm{H}^{3}(\mathrm{G}, \mathbb{Z})=\frac{\operatorname{Kerd}_{3}^{*}}{l m d_{2}^{*}}=\frac{\left\{\left(a_{2}, a_{2}, a_{3}, a_{4}\right) \mid l a_{2}=a_{4}, l a_{4}=0 ; a_{1}, a_{2}, a_{3}, a_{4} \in Z\right\}}{\left.\left\{0, l a_{2}, l a_{3}, 0\right) \mid a_{2}, a_{3} \in Z\right\}} \cong \mathbb{Z}_{l} \oplus \mathbb{Z}_{l}$

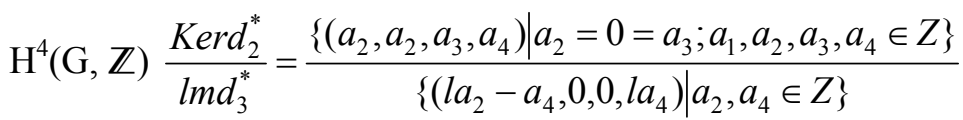

$$
\cong \frac{(x) \oplus(y)}{(l x,-x+l y)} \cong(x, y \mid l x=0, x=l y) \cong\left(y \mid l^{2}=0\right) \cong \mathbb{Z} l^{2} .
$$

Therefore $\mathrm{H}^{2 \mathrm{n}-1}(\mathrm{G}, \mathbb{Z}) \cong \mathbb{Z}_{l} \oplus \mathbb{Z}_{l}, \mathrm{n} \geq 2$.

$\mathrm{H} 2 \mathrm{n}(\mathrm{G}, \mathbb{Z}) \cong \mathbb{Z}_{l}^{2}, n \geq 1$.

\section{Determination of Homology and Cohomology of the Metacyclic Group}

An infinite Metacyclic group $M$ has a presentation:

generators : $h_{1}, h_{2}$;

relations : $h_{2}^{r}=1, h_{1} h_{2} h_{1}^{-1}=h_{2}^{t} ;(r, t)=1$.

Every finite metacyclic group can be regarded as a factor group of $M$.

We see that, $M=F / R$, where, $F$ is free group generated by $x_{1}, x_{2}$ (say) and $R$ is the normal subgroup of $F$ generated by $r_{1}, r_{2}$ where $r_{1}=x_{2}^{r}, r_{2}=x_{2}^{-t} x_{1} x_{2} x_{1}^{-1}$. Then the Fox derivatives are:

$$
\begin{array}{ll}
\frac{\partial r_{1}}{\partial x_{1}}=0 & \frac{\partial r_{1}}{\partial x_{2}}=x_{2}^{r-1}+\ldots \ldots+1, \\
\frac{\partial r_{2}}{\partial x_{1}}=x_{2} x_{1}^{-1}-x_{1}^{-1}, & \frac{\partial r_{2}}{\partial x_{2}}=-\left(x_{2}^{-t}+x_{2}^{-(t-1)}+\ldots \ldots \ldots+x_{2}^{-1}\right) \mathrm{x}_{1} \mathrm{x}_{2} x_{1}^{-1}+x_{1}^{-1}
\end{array}
$$

Let $\pi: \mathbb{Z} F \rightarrow \mathbb{Z} M$ be the homomorphism induced by the canonical homomorphism of $F$ onto $M$ with $R$ as the kernel. Let $\pi\left(x_{1}\right)=h_{1}, \pi\left(x_{2}\right)=h_{2}$. 


\section{Theorem 3.1}

The following is a free $\mathbb{Z} M$-resolution of $\mathbb{Z}$ :

$$
Y \stackrel{d_{4}}{\rightarrow} Y \stackrel{d_{3}}{\rightarrow} Y \stackrel{d_{2}}{\rightarrow} Y \stackrel{d_{1}}{\rightarrow} Y \stackrel{d_{0}}{\rightarrow} \mathbb{Z} \mathrm{M} \stackrel{\mathcal{E}}{\rightarrow} \mathrm{M} \rightarrow 0
$$

where $Y$ is a right $\mathbb{Z} M$-module free on $\alpha, \beta$ and $\varepsilon, d_{0}, d_{1}, d_{2}, d_{3} \ldots$ are the $\mathbb{Z} M$ homomorphisms given by

$$
\begin{aligned}
& \varepsilon(m)=1, \quad \text { for all } m \in M, \\
& d_{0}(\alpha)=h_{1}-1, \quad d_{0}(\beta)=h_{2}-1, \\
& d_{1}(\alpha)=\beta\left(h_{2}^{r-1}+\ldots \ldots+1\right), \\
& d_{1}(\beta)=\alpha\left(h_{2}-1\right) h_{1}^{-1}+\beta h_{1}^{-1}-h_{2}^{-t}\left(1+h_{2}+\cdots+h_{2}^{t-1}\right), \\
& d_{2}(\alpha)=\alpha\left(h_{2}-1\right), \\
& d_{2}(\beta)=\alpha\left(t-h_{1}^{-1}\right)+\beta\left(h_{2}^{t(r-1)}+\cdots+h_{2}^{t}+1\right), \\
& d_{3}(\alpha)=\alpha\left(h_{2}^{r-1}+\ldots+1\right), \\
& d_{3}(\beta)=\alpha\left(h_{1}^{-1}-t\left(h_{2}^{t-1}+\cdots+1\right)\right)+\beta\left(h_{2}^{t}-1\right), \\
& d_{4}(\alpha)=\alpha\left(h_{2}-1\right), \\
& d_{4}(\beta)=\alpha\left(t^{2}-h_{1}^{-1}\right)+\beta\left(h_{2}^{t(r-1)}+\cdots+h_{2}^{t}+1\right), \\
& d_{5}(\alpha)=\alpha\left(h_{2}^{r-1}+\ldots \ldots+1\right), \\
& d_{5}(\beta)=\alpha\left(h_{1}^{-1}-t^{2}\left(h_{2}^{t-1}+\cdots+1\right)\right)+\beta\left(h_{2}^{t}-1\right), \\
& d_{6}(\alpha)=\alpha\left(h_{2}-1\right), \\
& d_{6}(\beta)=\alpha\left(t^{3}-h_{1}^{-1}\right)+\beta\left(h_{2}^{t(r-1)}+\cdots+h_{2}^{t}+1\right), \text { and so on. }
\end{aligned}
$$

From the nature of these maps and the modules the sequence is clearly exact.

\section{Homology Groups of $M$}

Let $\mathrm{A}$ be a left $\mathbb{Z} M$-module, then homology groups $H_{n}(M, \mathbb{Z})$ are given by the homology of the complex:

$\ldots \ldots \ldots \ldots \rightarrow A^{2} \stackrel{\bar{d}_{4}}{\rightarrow} A^{2} \stackrel{\bar{d}_{3}}{\rightarrow} A^{2} \stackrel{\bar{d}_{2}}{\rightarrow} A^{2} \stackrel{\bar{d}_{1}}{\rightarrow} A^{2} \stackrel{\bar{d}_{0}}{\rightarrow} A \rightarrow 0$

where $A^{2}$ is written for $A \times A$, and the homomorphisms $\bar{d}_{0}, \bar{d}_{1}, \bar{d}_{2}, \bar{d}_{3}, \bar{d}_{4}, \ldots$, are induced by $d_{0}, d_{1}, d_{2}, d_{3}, d_{4}, \cdots$, respectively and are given by

$$
\bar{d}_{0}\left(a_{1}, a_{2}\right)=\left(h_{1}-1\right) a_{1}+\left(h_{2}-1\right) a_{2}
$$




$$
\begin{aligned}
& \bar{d}_{1}\left(a_{1}, a_{2}\right)=\left(\left(h_{2}-1\right) h_{1}^{-1} a_{2},\left(h_{2}^{r-1}+\cdots+1\right) a_{1}+h_{1}^{-1} a_{2}-\left(h_{2}^{t-1}+\cdots+1\right) a_{2}\right), \\
& \bar{d}_{2}\left(a_{1}, a_{2}\right)=\left(\left(h_{2}-1\right) a_{1}+\left(t-h_{1}^{-1}\right) a_{2},\left(h_{2}^{t(r-1)}+\cdots+h_{2}^{t}+1\right) a_{2}\right), \\
& \bar{d}_{3}\left(a_{1}, a_{2}\right)=\left(\left(h_{2}^{r-1}+\cdots+1\right) a_{1}+\left(h_{1}^{-1}-t\left(h_{2}^{t-1}+\cdots+1\right)\right) a_{2},\left(h_{2}^{t}-1\right) a_{2}\right), \\
& \bar{d}_{4}\left(a_{1}, a_{2}\right)=\left(\left(h_{2}-1\right) a_{1}+\left(t^{2}-h_{1}^{-1}\right) a_{2},\left(h_{2}^{t(r-1)}+\cdots+h_{2}^{t}+1\right) a_{2}\right), \\
& \bar{d}_{5}\left(a_{1}, a_{2}\right)=\left(\left(h_{2}^{r-1}+\cdots+1\right) a_{1}+\left(h_{1}^{-1}-t^{2}\left(h_{2}^{t-1}+\cdots+1\right)\right) a_{2},\left(h_{2}^{t}-1\right) a_{2}\right), \\
& \bar{d}_{6}\left(a_{1}, a_{2}\right)=\left(\left(h_{2}-1\right) a_{1}+\left(t^{3}-h_{1}^{-1}\right) a_{2},\left(h_{2}^{t(r-1)}+\cdots+h_{2}^{t}+1\right) a_{2}\right),
\end{aligned}
$$

When $A$ is a trivial $\mathbb{Z} M$-module $\mathbb{Z}$, the above homomorphisms reduce to the following:

$$
\begin{aligned}
& \bar{d}_{0}\left(a_{1}, a_{2}\right)=0, \\
& \bar{d}_{1}\left(a_{1}, a_{2}\right)=\left(0, r a_{1}+a_{2}-t a_{2}\right), \\
& \bar{d}_{2}\left(a_{1}, a_{2}\right)=\left((t-1) a_{2}, r a_{2}\right), \\
& \bar{d}_{3}\left(a_{1}, a_{2}\right)=\left(r a_{1}+a_{2}-t^{2} a_{2}, 0\right), \\
& \bar{d}_{4}\left(a_{1}, a_{2}\right)=\left(\left(t^{2}-1\right) a_{2}, r a_{2}\right), \\
& \bar{d}_{5}\left(a_{1}, a_{2}\right)=\left(r a_{1}+a_{2}-t^{3} a_{2}, 0\right), \\
& \bar{d}_{6}\left(a_{1}, a_{2}\right)=\left(\left(t^{3}-1\right) a_{2}, r a_{2}\right),
\end{aligned}
$$

Therefore, in this case, we have

$H_{0}(M, \mathbb{Z}) \cong \mathbb{Z}$

$\mathrm{H}_{1}(M, \mathbb{Z})=\frac{\operatorname{Ker} \bar{d}_{0}}{\operatorname{lm} \bar{d}_{1}}=\frac{\left\{\left(a_{1}, a_{2}\right) \mid a_{1}, a_{2} \in Z\right\}}{\left\{\left(0, r a_{2}+(1-t) a_{2}\right) \mid a_{1}, a_{2} \in Z\right\}} \cong \mathbb{Z} \oplus \mathbb{Z}_{h}(2)$ where $h^{(1)}=h . c . f .(r, 1-t)$

$\mathrm{H}_{2}(M, \mathbb{Z})=\frac{\operatorname{Ker} \bar{d}_{1}}{\operatorname{lm} \bar{d}_{2}}=\frac{\left\{\left(a_{1}, a_{2}\right) \mid r a_{2}+(1-t) a_{2}=0 ; a_{1}, a_{2} \in Z\right\}}{\left\{\left((t-1) a_{1}, r a_{2}\right) \mid a_{1}, a_{2} \in Z\right\}} \cong \frac{Z_{\infty}(s, r)}{Z_{\infty}\left(h^{(2)}(s, r)\right)} \cong \mathbb{Z}_{h}(2)$.

$\mathrm{H}_{3}(M, \mathbb{Z})=\frac{\operatorname{Ker} \bar{d}_{2}}{\operatorname{lm} \bar{d}_{3}}=\frac{\left\{\left(a_{1}, a_{2}\right) \mid(t-1) a_{1}=0=r a_{2} ; a_{1}, a_{2} \in Z\right\}}{\left\{\left(r a_{1}+\left(1-t^{2}\right) a_{2}, 0\right) \mid a_{1}, a_{2} \in Z\right\}} \cong \mathbb{Z}_{h}^{(2)}$.

Where $h^{(2)}=\left(r, 1-t^{2}\right), r=h^{(2)} r^{\prime \prime}, 1-t^{2}=h^{(2)} s^{\prime \prime}$. 
$\mathrm{H}_{4}(M, \mathbb{Z})=\frac{\operatorname{Ker} \bar{d}_{3}}{\operatorname{lm} \bar{d}_{4}}=\frac{\left\{\left(a_{1}, a_{2}\right) \mid r a_{1}+\left(1-t^{2}\right) a_{2}=0 ; a_{1}, a_{2} \in Z\right\}}{\left\{\left(\left(t^{2}-1\right) a_{1}, r a_{2}\right) \mid a_{1}, a_{2} \in Z\right\}} \cong \frac{(s, r)}{\left(h^{(2)}(s, r)\right)} \cong \mathbb{Z}_{h}^{(2)}$.

$\mathrm{H}_{5}(M, \mathbb{Z})=\frac{\operatorname{Ker} \bar{d}_{4}}{\operatorname{lm} \bar{d}_{5}}=\frac{\left\{\left(a_{1}, a_{2}\right) \mid\left(t^{3}-1\right) a_{1}=0=r a_{2} ; a_{1}, a_{2} \in Z\right\}}{\left\{\left(r a_{1}+\left(1-t^{3}\right) a_{2}, 0\right) \mid a_{1}, a_{2} \in Z\right\}} \cong \frac{(1,0)}{\left(h^{(3)}(1,0)\right)} \cong \mathbb{Z}_{h}^{(3)}$,

where $h^{(3)}=\left(r, 1-t^{3}\right), r=h^{(3)} \mathrm{r}^{\prime \prime \prime}, 1-\mathrm{t}^{3}=\mathrm{h}^{(3)} \mathrm{s}^{\prime \prime \prime}$, since $1=\mathrm{r}^{\prime \prime \prime} \mathrm{a}_{1}+\mathrm{s}^{\prime \prime \prime} \mathrm{a}_{2}$ for some $\mathrm{a}_{1}, \mathrm{a}_{2} \in \mathbb{Z}$.

From the nature of the homomorphisms $H_{6}(M, \mathbb{Z}) \cong \mathbb{Z}_{h}(3)$.

Thus, in general, $H_{i}(M, \mathbb{Z}) \cong \mathbb{Z}_{h}(n), i=2 n-1,2 n, n>1$ and $h^{(n)}=\left(r, 1-t^{n}\right)$.

\section{Cohomology Groups of $M$}

Let $\mathrm{A}$ be a right $\mathbb{Z} M$-module, then the cohomology groups $H^{n}(M, A)$ are given by the homology of the complex:

$$
\leftarrow A^{2} \stackrel{d_{6}^{*}}{\leftarrow} A^{2} \stackrel{d_{5}^{*}}{\leftarrow} A^{2} \stackrel{d_{4}^{*}}{\leftarrow} A^{2} \stackrel{d_{3}^{*}}{\leftarrow} A^{2} \stackrel{d_{2}^{*}}{\leftarrow} A^{2} \stackrel{d_{2}^{*}}{\leftarrow} A^{2} \stackrel{d_{0}^{*}}{\leftarrow A} A \leftarrow 0
$$

where the homomorphisms $d_{0}^{*}, d_{1}^{*}, d_{2}^{*}, d_{3}^{*}, \cdots$ are induced by $d_{0}, d_{1}, d_{2}, d_{3}, \cdots$

respectively and given by

$$
\begin{aligned}
& d_{0}^{*}(a)=\left(a\left(h_{1}-1\right), a\left(h_{2}-1\right)\right), \\
& d_{1}^{*}\left(a_{1}, a_{2}\right)=\left(a_{2}\left(h_{2}^{r-1}+\cdots+1\right), a_{1}\left(h_{2}-1\right) h_{1}^{-1}+a_{2} h_{1}^{-1}-a_{2}\left(h_{2}^{t-1}+\cdots+1\right)\right), \\
& d_{2}^{*}\left(a_{1}, a_{2}\right)=\left(a_{1}\left(h_{2}-1\right), a_{1}\left(t-h_{1}^{-1}\right)+a_{2}\left(h_{2}^{t(r-1)}+\cdots+h_{2}^{t}+1\right)\right) \\
& d_{3}^{*}\left(a_{1}, a_{2}\right)=\left(a_{1}\left(h_{2}^{r-1}+\cdots+1\right), a_{1}\left(h_{1}^{-1}-t\left(h_{2}^{t-1}+\cdots+1\right)\right)+a_{2}\left(h_{2}^{t}-1\right)\right), \\
& d_{4}^{*}\left(a_{1}, a_{2}\right)=\left(a_{1}\left(h_{2}-1\right), a_{1}\left(t^{2}-h_{1}^{-1}\right)+a_{2}\left(h_{2}^{t(r-1)}+\cdots+h_{2}^{t}+1\right)\right), \\
& d_{5}^{*}\left(a_{1}, a_{2}\right)=\left(a_{1}\left(h_{2}^{r-1}+\cdots+1\right), a_{1}\left(h_{1}^{-1}-t^{2}\left(h_{2}^{t-1}+\cdots+1\right)\right)+a_{2}\left(h_{2}^{t}-1\right)\right), \\
& d_{6}^{*}\left(a_{1}, a_{2}\right)=\left(a_{1}\left(h_{2}-1\right), a_{1}\left(t^{3}-h_{1}^{-1}\right)+a_{2}\left(h_{2}^{t(r-1)}+\cdots+h_{2}^{t}+1\right)\right),
\end{aligned}
$$

and so on, for all $a, a_{1}, a_{2} \in A$.

When $A$ is a trivial $\mathbb{Z} M$-module $\mathbb{Z}$, the above homomorphisms reduce to the following:

$$
\begin{aligned}
& d_{0}^{*}(a)=(0,0), \\
& d_{1}^{*}\left(a_{1}, a_{2}\right)=\left(r a_{2}, a_{2}-t a_{2}\right), \\
& d_{2}^{*}\left(a_{1}, a_{2}\right)=\left(0, r a_{2}+a_{1}(t-1)\right),
\end{aligned}
$$




$$
\begin{aligned}
& d_{3}^{*}\left(a_{1}, a_{2}\right)=\left(r a_{1}, a_{1}\left(1-t^{2}\right)\right), \\
& d_{4}^{*}\left(a_{1}, a_{2}\right)=\left(0, a_{1}\left(t^{2}-1\right)+r a_{2}\right), \\
& d_{5}^{*}\left(a_{1}, a_{2}\right)=\left(r a_{1}, a_{1}\left(1-t^{3}\right)\right), \\
& d_{6}^{*}\left(a_{1}, a_{2}\right)=\left(0, a_{1}\left(t^{3}-1\right)+r a_{2}\right) .
\end{aligned}
$$

Therefore, in that case, we have

$\mathrm{H}^{0}(M, \mathbb{Z}) \cong \mathbb{Z}$

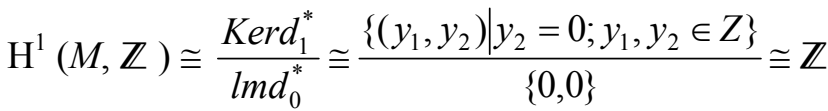

$\mathrm{H}^{2}(M, \mathbb{Z}) \cong \frac{\operatorname{Kerd}_{2}^{*}}{l m d_{1}^{*}} \cong \frac{\left\{\left(y_{1}, y_{2}\right) \mid r y_{2}+(t-1) y_{1}=0 ; y_{1}, y_{2} \in Z\right\}}{\left\{\left(r y_{1},(1-t) y_{2}\right) \mid y 1, y_{2} \in Z\right\}} \cong \frac{Z_{\infty}(r, s)}{Z_{\infty}\left(h^{(1)}\left(r^{\prime}, s^{\prime}\right)\right)} \cong \mathbb{Z}_{h}^{(1)}$.

$\mathrm{H}_{3}(M, \mathbb{Z}) \cong \frac{\operatorname{Ker} \bar{d}_{2}}{\operatorname{lm} \bar{d}_{3}}=\frac{\left\{\left(y_{2}, y_{2}\right) \mid r y_{2}=0=\left(1-t^{2}\right) y_{2}=0 ; y_{2}, y_{2} \in Z\right\}}{\left\{\left(r y_{2}+(1-t) y_{1}\right) \mid y_{2}, y_{2} \in Z\right\}} \cong \frac{\left\{\left(0, y_{2}\right)\right\}}{\left\{\left(0,\left(\left(r y_{2}+s y_{2}\right) h^{(1)}\right)\right.\right.}$

$\cong \mathbb{Z}_{h}^{(1)}$.

where $h^{(1)}=(r, 1-t), 1=r^{\prime} y_{2}+s^{\prime} y_{1}$.

$\mathrm{H}_{4}(M, \mathbb{Z}) \cong \frac{\operatorname{Ker} \bar{d}_{4}}{\operatorname{lm} \bar{d}_{3}}=\frac{\left\{\left(y_{1}, y_{2}\right) \mid r y_{1}+\left(t^{2}-1\right) y_{2}=0 ; y_{1}, y_{2} \in Z\right\}}{\left\{\left(r y_{1},\left(1-t^{2}\right) y_{1}\right) \mid y_{1}, y_{2} \in Z\right\}} \cong \frac{Z_{\infty}(r, s)}{Z_{\infty}\left(h^{(2)}\left(r^{\prime \prime}, s^{\prime \prime}\right)\right)} \cong \mathbb{Z}_{h}^{(2)}$.

$\mathrm{H}_{5}(M, \mathbb{Z}) \cong \frac{\operatorname{Ker} \bar{d}_{5}}{\operatorname{lm} \bar{d}_{4}}=\frac{\left\{\left(y_{1}, y_{2}\right) \mid r y_{1}=0=\left(1-t^{3}\right) y_{2} ; y_{1}, y_{2} \in Z\right\}}{\left\{\left(0, r y_{1}+\left(t^{2}-1\right) y_{1}\right) \mid y_{1}, y_{2} \in Z\right\}} \cong \frac{\left\{\left(0, y_{2}\right)\right\}}{\left\{\left(0,\left(r^{\prime} y_{2}+s^{\prime \prime} y^{2}\right) h^{(2)}\right)\right.} \cong \mathbb{Z}_{h}^{(2)}$.

where $h^{(2)}=\left(r, 1-t^{2}\right), 1=r^{\prime \prime} y_{2}+s^{\prime \prime} y_{1}$.

$\mathrm{H}^{6}(M, \mathbb{Z}) \cong \frac{\operatorname{Ker} \bar{d}_{6}}{\operatorname{lm} \bar{d}_{5}}=\frac{\left\{\left(y_{1}, y_{2}\right) \mid r y_{1}+\left(t^{3}-1\right) y_{2}=0 ; y_{1}, y_{2} \in Z\right\}}{\left\{\left(r y_{1},\left(1-t^{3}\right) y_{2}\right) \mid y_{1}, y_{2} \in Z\right\}} \cong \frac{Z_{\infty}(r, s)}{Z_{\infty}\left(h^{(3)}\left(r^{\prime \prime \prime}, s^{\prime \prime \prime}\right)\right)} \cong \mathbb{Z}_{h}^{(3)}$.

Thus in general, $H^{i}(M, \mathbb{Z}) \cong \mathbb{Z}_{h}^{(\mathrm{n})}$, for $\mathrm{i}=2 \mathrm{n}, 2 \mathrm{n}+1, \mathrm{n} \geq 1$ and $h^{(n)}=\left(\mathrm{r}, 1-t^{n}\right)$.

\section{REFERENCES}

[1] Akhter, N. and Majumdar, S.; Determination of the homology and the cohomology of NEC groups with non-orientable orbit spaces and no reflexions, Bull. Cal. Math. Soc., 95(3), 2003, 207-218.

[2] Carbone, L.J., A filtration of the chain complex of rewriting system, DIMACS Series in Discrete Math.and Theoretical Computer Science Vol.25, 1996.

[3] Cartan, H. and Eilenberg, S: Homological algebra (Oxford University Press), 1956.

[4] Fox, R.H., Free Differential Calculus I, Ann. Math. 57, 3, 1953, p. 547-560. 
[5] Grove, J.R.J., Rewriting systems and homology of groups, Proc. of the third International Conference on the Theory of Groups, Canberra, (1456 LNM, Springer-Verlag), 1989.

[6] Gruenberg, K.W. Resolutions by relations, J. of London Math. Soc., 35, 1960, 481-494.

[7] Gruenberg, K.W.,Cohomological topics in group theory, Spinger-Verlag, New York, 1970.

[8] Huebschmann, J.,Cohomology theory of aspherical groups and of small can- cellation groups, J. Pure Appl. Algebra, 14, 1979,137-143.

[9] Huebschmann, J., Perturbation theory and free resolutions for nilpotent groups of class 2, J. of Algebra, $126,2,1989,348-399$.

[10] Huebschmann, J., Cohomology of nilpotent groups of class 2, J. of Algebra 126, 1989, 400-450.

[11] Huebschmann, J. The mod-p cohomology rings of metacyclic groups, J. of Pure Appl. Algebra 60, 1989, 53-103.

[12] Learner, A.,Cohomology of groups, Lecture notes, Queen Mary College, University of London, 1965.

[13] Lyndon, R.C.,Cohomology theory of groups with a single defining relation, Ann. of Math. 52, 3, 1950, 656-665.

[14] Majumdar, S., A free resolution for a class of groups, J. Lond. Math. Soc., 2, 2, 1970, 615-619.

[15] Majumdar, S., A free resolution for a general class of groups, Tech. Report Math.Stat. (17) 17, 1971, ISI, Calcutta.

[16] Majumdar, S., Free resolutions for certain classes of groups, Proc. Edin. Math. Soc. 26, 1983, 173-179.

[17] Majumdar, S., Homology and cohomology of a class of polycyclic groups, Internal report, IC/84/177, 1984, ICTP, Trieste, Italy.

[18] Majumdar, S.,On the homology and cohomology of certain polycyclic groups, Internal report, IC/87/305,1987, ICTP, Trieste, Italy.

[19] Majumdar, S. and Akhter, N.: On a simple method of determining the homology and the cohomology for finitely presented groups, J. Bang. Acad. Sci. Vol. 34, No. 2, 2010, 103-114.

[20] Majumdar, S. and Akhter, N., Determination of the homology and the cohomology of the trifoil knot group and the fundamental group of an interesting manifold N ,Ganit: J. Bangladesh, Math. Soc., 22, 2002, 7-14.

[21] Majumdar, S. and Akhter, N., Determination of the homology and the cohomology of a class of infinite groups associated with groups of Fibonacci type,Bull. Cal. Math. Soc., 2008, 100.

[22] Scott, P., The geometries 3- manifolds, Bull. Lond. Math. Soc. Vol. 15, No. 56, 1983, 401-487. 